\section{MELANOMA}

\section{Stripy surrogates}

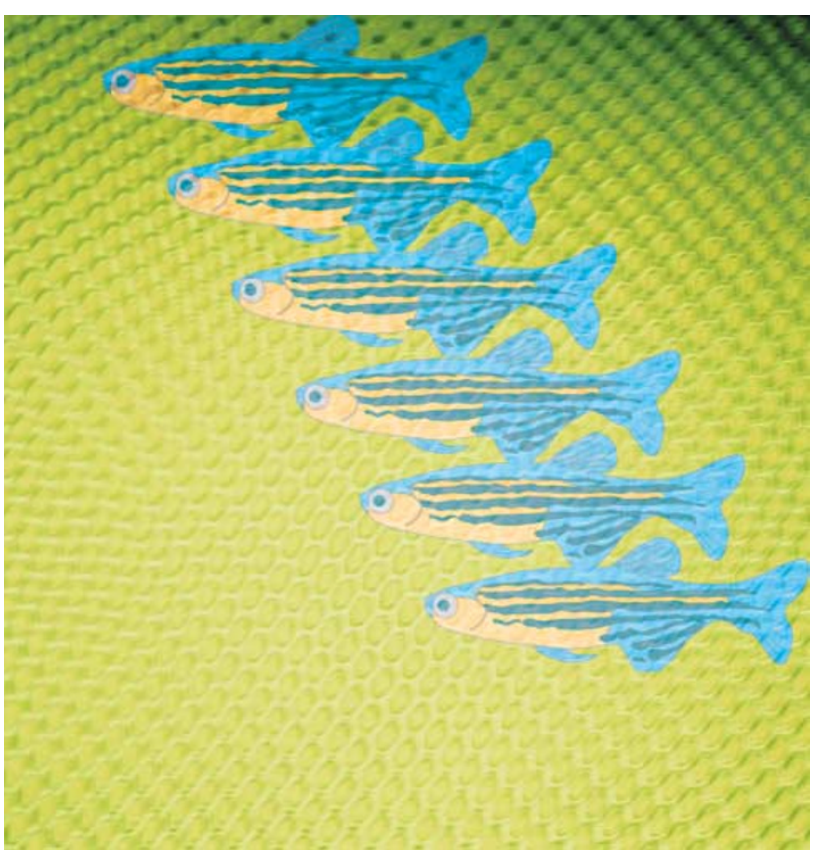

Most human melanomas have mutations in the serine/threonine kinase BRAF, but the role of this enzyme in tumorigenesis is not well understood, partly because there has been no animal model with the relevant mutations. E. Elizabeth Patton and colleagues have used zebrafish to show that BRAF interacts genetically with the p53 pathway to cause melanomas.

The zebrafish's characteristic stripes are created by melanin, and the fish's melanin-producing cells are remarkably similar to melanocytes in mammals - they derive from the same embryonic lineage (the neural crest) and at least one of the genes controlling melanocyte differentiation is homologous in mice and zebrafish. Patton and colleagues surmised that if the normal growth and development of melanocytes is evolutionarily conserved among vertebrates, then their dysfunctional growth might be too.

They microinjected zebrafish embryos at the one-cell stage with the human BRAF gene containing the most common activating mutation (V600E) under the control of the melanocyte-specific mitfa promoter. About $10 \%$ of the resulting fish developed mole-like foci of proliferating melanocytes, termed f-nevi. The melanocytes in the f-nevi were well differentiated and appeared not to invade the surrounding tissue. So although the activating $B R A F^{\mathrm{V} 600 \mathrm{E}}$ mutation is sufficient to cause expansion of the melanocytes, further mutations seem to be required for progression to melanoma.

Human melanomas often lack the $C D K N 2 A$ locus, in addition to mutation of BRAF. This locus encodes the tumour-suppressor protein ARF, which functions in the p53 pathway. Although p53 mutations are rare in melanomas, inactivation of this pathway has been linked to their formation. Patton and colleagues speculated that a deficiency in the p53 pathway might be the extra push that drives BRAFmutated melanocytes into forming invasive tumours.

\title{
Small size, big potential
}

Human liver-receptor homologue 1 (LRH1) has a crucial role in the progression of homone-sensitive breast cancer. A recent analysis of its stucture and functional interactions has identified LRH1 as a possible target for small-molecule inhibitors that could potentially slow the progression of this disease.

LRH1 is a nuclear receptor that, both directly and indirectly, controls the expression of several genes, including those involved in cholesterol and lipid metabolism. Particularly interesting is its control of genes that are involved in hormone synthesis, especially CYP19, which encodes an oestrogen-synthesis enzyme (aromatase) that is expressed in the pre-adipocytes of breast carcinomas.

LRH1 can interact with various common transcriptional co-regulators, although a particular part of its structure, known as AF-2, is not optimized for these interactions. However, small heterodimer partner (SHP) can interact with LRH1 efficiently, and represses its activity in many tissues. Ortlund et al. therefore set out to clarify the structural basis for LRH1-mediated transcriptional control and for the control of LRH1 activity by SHP.

Surprisingly, analysis of the ligandbinding domain of LRH1 showed that it binds the phospholipids phosphatidylethanolamine and phosphatidylglycerol. In addition, various features of the nuclear receptor box 1 motif of SHP (which binds a groove on the surface of LRH1) were identified that explain the 'preference' of LRH1 for SHP and for certain other nuclear co-regulators that include DAX1 and PGC1 $\alpha$. Structural analysis of helix 12 (H12) in the AF-2 region of LRH1 also showed that a series of contacts between SHP and LRH1 'rigidify' the position of $\mathrm{H} 12$, which reduces the affinity of LRH1 for common co-repressors and enhances its affinity for SHP, DAX1 and PGC1 $\alpha$. Significantly, the interaction of LRH1 with PGC1 $\alpha$ is crucial for CYP19 expression, and thereby oestrogen generation, which drives local tumour growth in breast cancer.

The authors then showed that obscuring the lipid-binding pocket of LRH1 by mutating two amino-acid residues near the pocket entrance reduced the level of phospholipid binding by LRH1 and significantly lowered its transcriptional activity. Moreover, functional studies, including glutathione- $S$-transferase pull-down and mammalian two-hybrid experiments, further clarified that the amount of phospholipid that is bound to LRH1 influences its activity in mammalian cells.

So, along with previous studies, these results indicate that phospholipids can have important roles in transcriptional regulation. Furthermore, the demonstration that LRH1 is regulated by ligands makes it a candidate target for small-molecule inhibitors. Given the crucial role of LRH1 in breast cancer development, such inhibitors could be useful in breast cancer therapy.

(i) References and links Lesley Cunliffe ORIGINAL RESEARCH PAPER Ortlund, E. A. et al. Modulation of human nuclear receptor $\mathrm{LRH}-1$ activity by phospholipids and SHP. Nature Struct. Mol. Biol. 22 Feb 2005 (doi:10.1038/nsmb910) 
Injecting mitfa- $B R A F^{\mathrm{V} 600 \mathrm{E}}$ into embryos homozygous for a mutation in TP53 resulted in about $6 \%$ of the fish developing malignant melanomas by 4 months of age. The melanoma cells expressed melanin and were morphologically similar to human melanoma cells. The cells spread rapidly following transplant into wildtype zebrafish (unlike cells from the f-nevi), and $89 \%$ of them showed chromosomal abnormalities similar to those seen in human melanomas.

With this zebrafish model of melanoma, Patton et al. demonstrate that it is feasible to use these animals to explore the genetic interactions that cause malignant phenotypes, and provide evidence that BRAF mutations do indeed underlie this disease.

Helen Dell

\section{(2) References and links}

ORIGINAL RESEARCH PAPER Patton, E. E. et al. BRAF mutations are sufficient to promote nevi formation and cooperate with $\mathrm{p} 53$ in the genesis of melanoma. Curr. Biol. 15, 249-254 (2005) FURTHER READING Chin, L. The genetics of malignant melanoma: from mouse to man. Nature Rev. Cancer 3, 559-570 (2003)

WEB SITE

Leonard Zon's lab: http://Zon.tchlab.org/

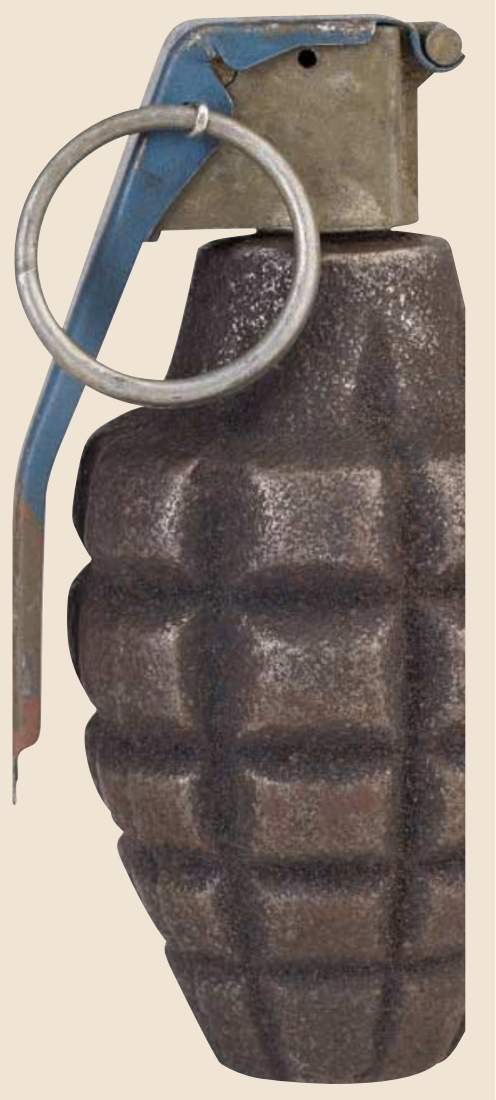

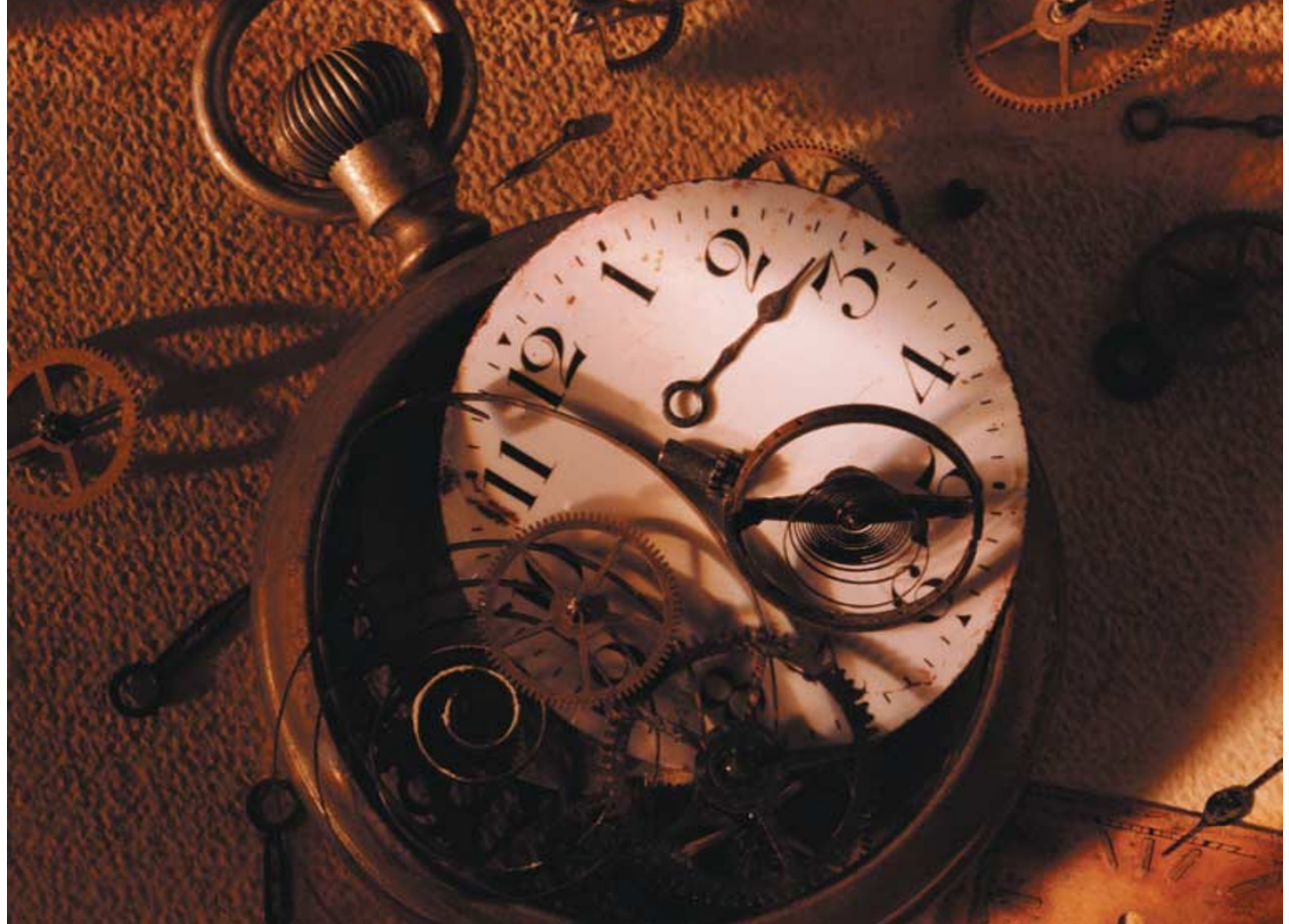

METASTASIS

\section{Moving PARts}

Protease-activated receptors (PARs) are

G-protein-coupled receptors that act as sensors of extracellular protease gradients, allowing cells to react to the proteolytic microenvironment during tissue remodelling in development, inflammation and angiogenesis, and also in cancer. Athan Kuliopulos and colleagues set out to investigate the role of PARs during cancer invasion and metastasis, and, in doing so, have uncovered a new PAR protease and a novel link between invading cells and the extracellular matrix.

The authors focused on PAR1, an oncogene that has long been thought to be involved in the invasion and metastasis of melanomas and cancers of the breast, colon, lung, pancreas and prostate. PAR1 responds to a select group of serine proteases that includes thrombin, plasmin, $\mathrm{Xa}$ and activated protein $\mathrm{C}$. These cleave the PAR1 extracellular domain and induce transmembrane signalling, but, curiously, none of these proteases are crucial for the invasive properties of PAR1dependent breast cancer cells.

Kuliopulos and colleagues started by investigating the role of PAR1 during the invasive interaction between breast cancer cells and the stroma. They showed that the migration of breast cancer cells towards fibroblast-derived media does require 'proteolysable' PAR1; cells transfected with 'proteolytically dead' PAR1 are unable to migrate.

The authors then confirmed that PAR 1 is a highly tumorigenic and invasogenic factor in vivo, by transfecting MCF-7 cells - which resemble early breast cancer cells - with PAR1 and injecting them into the mammary fat pads of nude mice. They also carried out the converse experiment, using small interfering RNA to knock down levels of PAR1 in the highly tumorigenic cell line MDA-MB-231, markedly reducing migration and invasion. So if PAR1 confers invasive and migration properties on breast cancer cells, which protease is activating it?

The authors tested a range of proteases and protease inhibitors for their ability to affect the migration of PAR1-expressing breast cancer cells in vitro. To their surprise, the only stromal protease that was found to induce migration was matrix metalloproteinase 1 (MMP1). They went on to show that MMP1 that is secreted by fibroblasts cleaves PAR1, providing the first demonstration of direct activation of a G-proteincoupled receptor by an MMP. It has previously been shown that MMPs are expressed by stromal fibroblasts and inflammatory cells that are recruited to tumours, and MMP1 is a marker of poor prognosis in breast, colorectal and oesophageal tumours. These results, together with the findings of Kuliopulos and co-workers, indicate that breast cancer cells can use fibroblastderived MMP1 and PAR1 as a chemotactic signal to invade surrounding stromal tissues.

The researchers note that several MMP inhibitors have been tested in Phase III clinical trials to treat diverse cancers, but patients have suffered from joint toxicity that is thought to be due to MMP1 inhibition. Their investigations of PAR1 or MMP1 as therapeutic targets showed that inhibiting either target in vivo substantially reduced tumour growth, invasion and vascularity, so PAR1 could be used as a downstream target for therapies that aim to block MMP1.

Jenny Bangham

(2) References and links ORIGINAL RESEARCH PAPER Boire, A. et al. PAR1 is a matrix metalloprotease- 1 receptor that promotes invasion and tumorigenesis of breast cancer cells. Cell 120, 303-313 (2005) 